\title{
STATISTICS OF EARTHQUAKE RECURRENCE TIME IN NORTH AEGEAN TROUGH
}

\author{
Kourouklas Ch. ${ }^{1}$, Papadimitriou E. ${ }^{1}$, Tsaklidis G. ${ }^{2}$ and Karakostas V.1 \\ ${ }^{1}$ Aristotle University of Thessaloniki, Department of Geology, 54124, Thessaloniki, Greece, \\ kouroukl@geo.auth.gr,ritsa@geo.auth.gr,vkarak@geo.auth.gr \\ ${ }^{2}$ Aristotle University of Thessaloniki, School of Mathematics, 54124, Thessaloniki, Greece, \\ tsaklidi@math.auth.gr
}

\begin{abstract}
The determination of recurrence time of strong earthquakes of certain magnitude on a specific fault or fault segment is an important component of seismic hazard assessment. The occurrence of these earthquakes is neither periodic nor completely random but often clustered in time. This fact in connection with their limited number inhibits a deterministic approach for recurrence times calculation and thus application of stochastic processes is required. For recurrence times determination in the area of North Aegean Trough, all the available information on strong earthquakes (historical and instrumental) with $M \geq 6.0$ is collected. Given that source parameters of historical events contain larger uncertainties, reassessment of their focal parameters before the application of stochastic processes is necessary, which was performed by applying the method of Bakun and Wentworth (1997). The reasses sed catalogue was divided into three data sets, according to the strong events spatial distribution and their association with distinctive fault segments. Three statistical dis tributions (Weibull, inverse Gaussian, lognormal) were applied and evaluated with $t$ he Anderson-Darling test and the Akaike and Bayesian Information Criteria. The $W$ eibull distribution exhibited better performance in two out of three data sets and the Inverse Gaussian distribution in the third. With given distributions the occurrence pr obabilities were calculated for strong events above a certain magnitude and for cert ain time interval.
\end{abstract}

Keywords: relocation of historical earthquakes, goodness of fit test, information criteria, earthquake probabilities.

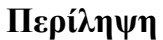

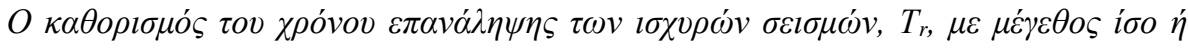

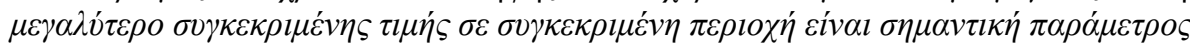

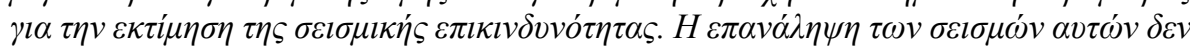

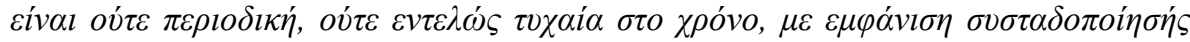

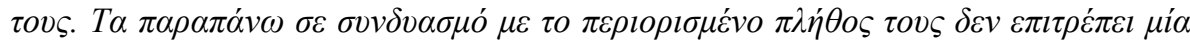

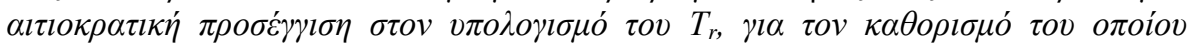

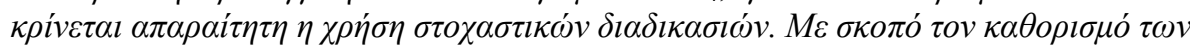

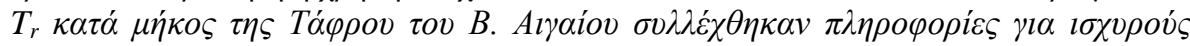

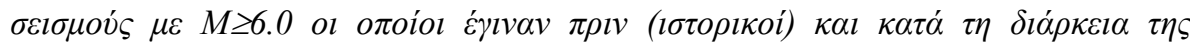

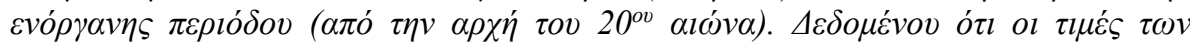

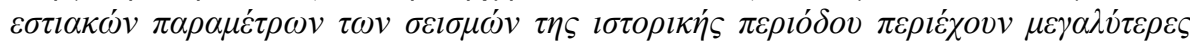




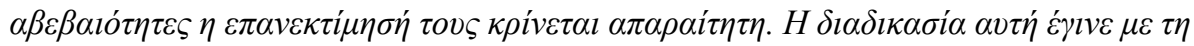

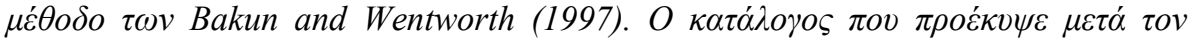

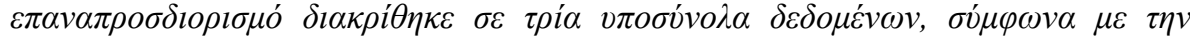

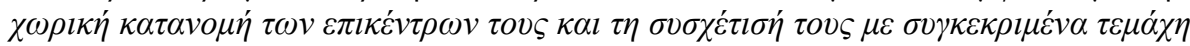

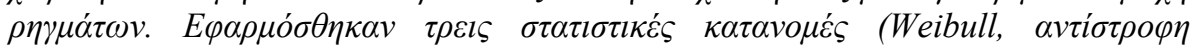

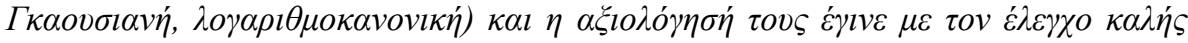

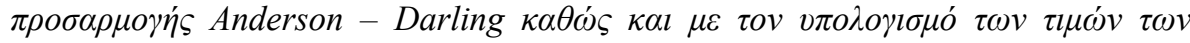

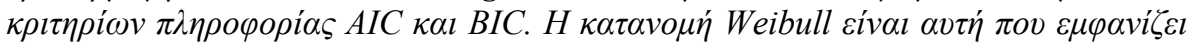

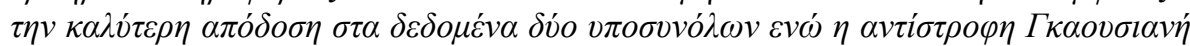

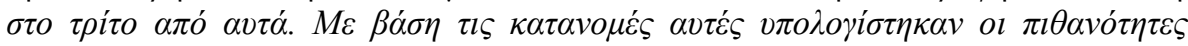

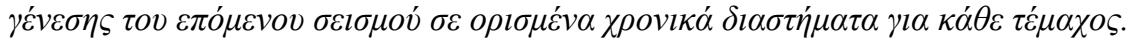

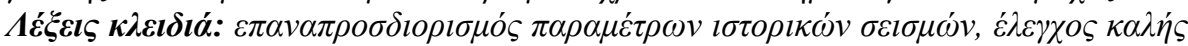

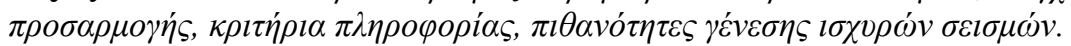

\section{Introduction}

The determination of recurrence time of strong earthquakes above a certain magnitude and on a specific fault or fault segment is an important factor for seismic hazard assessment. This determination is based on the time predictable model of earthquake occurrence (Shimazaki and Nakata, 1980) and the hypothesis of characteristic earthquake (Schwartz and Coppersmith, 1984). According to the time predictable model an earthquake occurs when stress exceeds a certain value, which is constant for every earthquake. Consequently, the time of the next earthquake can be estimated taking into account the coseismic slip of the previous one. The characteristic earthquake hypothesis assumes that strong earthquakes on a specific fault occur with similar magnitudes, similar rupture areas and within time intervals exhibiting some kind of regularity.

The occurrence of strong earthquakes, is neither periodic nor completely random but often clustered in time. In addition, the limitation in time of earthquake record hampers a deterministic calculation of the earthquake recurrence time. Consequently, the application of stochastic processes is required for this estimation with distributions such as Weibull (Hagiwara, 1974; Rikitake, 1976; Abaimov et al., 2008), Lognormal (Nishenko and Buland, 1987; Jackson et al. 1995) and Brownian Passage Time or Inverse Gaussian (Kagan and Knopoff, 1987; Matthews et al., 2002). Statistical processing requires a number of strong earthquakes in a specific region adequate for specific applications, which is often if not always limited. On the other hand, the interevent times exceed the instrumental period. Therefore, use of data both from historical and instrumental seismicity is necessary.

Since historical seismicity contains uncertainties, focal parameters reassessment of earthquake source parameters is performed. Numerical methods such as the one proposed by Bakun and Wentworth (1997) are widely used and this latter is engaged in our study for improving earthquake source parameters and consequently associate them with specific faults or fault segments.

The study area comprises the North Aegean Trough (NAT), which is among the most active ones in the Greek territory exhibiting frequent occurrence of strong earthquakes (Fig. 1). It constitutes the northern boundary of the south Aegean plate (Papazachos et al., 1998) and is the prolongation of the North Anatolian Fault (NAF) to the west, dominated by right - lateral strike - slip faulting. After relocation of historical earthquakes, the interevent times on certain fault segments were estimated with the ultimate goal being the evaluation of the next such event occurrence time onto each fault segment. 


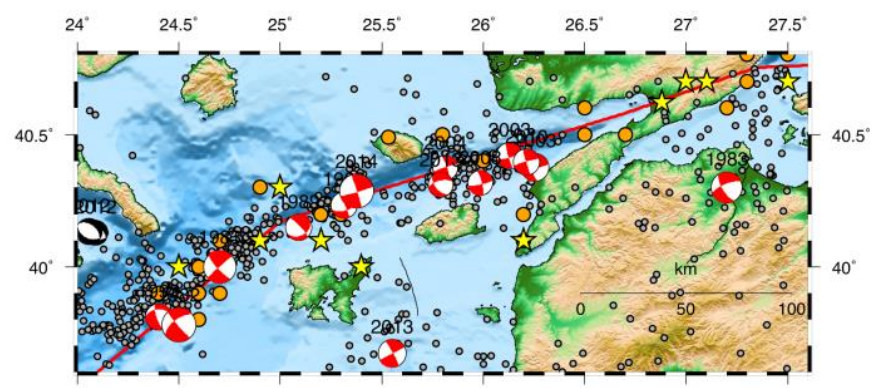

Figure 1 - Instrumental and historical seismicity along the North Aegean Trough (NAT). Small circles depict earthquakes with $M \geq 4.0$ from 1980, moderate circles all known with $M \geq 6.0$ and asterisks all known with $M \geq 7.0$. Fault plane solutions of $M \geq 5.0$ earthquakes available from gcmt solutions (http:/globalcmt.org) are plotted as equal area lower hemisphere projections.

\section{Methods}

\subsection{Reassessment of historical earthquakes}

The method of Bakun and Wentworth (1997) is used for the reassessment of the source parameters of historical events. It is a numerical method that uses the macroseismic intensities given in Modified Mercalli Intensity (MMI) scale and an attenuation relation for the study area. Calculation is achieved of the epicentre, the magnitude (equivalent with moment magnitude) and the corresponding error given by the root mean square (rms) by creating a grid of points onto the search area according with the relations:

\section{Equation 1 - Magnitude of earthquake}

$\boldsymbol{M}=\boldsymbol{M}_{\boldsymbol{I}}=\boldsymbol{e} \boldsymbol{a n}\left(\boldsymbol{M}_{\boldsymbol{i}}\right)$, where $M_{i}$ is the magnitude at the potential epicentre (i.e. the gridpoint $I$ ) inferred from each MMI value and an attenuation relation.

Equation 2 - rms calculation for each point of the grid

$r m s\left[M_{I}\right]=r m s\left[M_{I}-M_{i}\right]-r m s_{0}\left[M_{I}-M_{i}\right]$, where $r m s_{0}$ is the minimum value of $r m s$ for the whole grid.

\section{Equation 3 - Relation for the $\operatorname{rms}\left[\mathrm{M}_{\mathrm{I}}-\mathrm{M}_{\mathrm{i}}\right]$ factor}

$$
r m s\left[M_{I}-M_{i}\right]=\left\{\sum_{i}\left[w_{i}\left(M_{I}-M_{i}\right)\right]^{2} / \sum_{i} w_{i}{ }^{2}\right\}^{1 / 2}
$$

\section{Equation 4 - Distance - weighting function}

$w_{i}=\left\{\begin{array}{c}0.1+\cos \left[\left(\Delta_{i} / 150\right)(\pi / 2)\right], \text { for } \Delta_{i} \leq 150 \mathrm{~km} \\ 0.1, \text { for } \Delta_{i}>150 \mathrm{~km}\end{array}\right.$, where $\quad i$ is the distance (in $\left.\mathrm{km}\right)$ of observations $i$ from the assumed gridpoint $I$.

After combining these four relations, the method returns a grid of points, which represent the trial earthquake epicentres. The point with the least rsm is the most reliable point for the earthquake location. Four parameters are required for starting creating the grid of points, the longitude and the latitude of the center of the grid, the radius of the search area in $\mathrm{km}$ and the grid search spacing in $\mathrm{km}$. In this study, the attenuation relation of Papazachos and Papaioannou (1997) is used:

\section{Equation 5 - Attenuation relation}

$M=0.62 I+2.035 \log R+0.002 R-0.78$, where $\mathrm{R}=\left(\Delta^{2}-\mathrm{h}^{2}\right)^{1 / 2}$ is the hypocentral distance. 
A verification test of the method was performed on instrumental earthquakes for which macroseismic intensities were available and comparison is made of the results with those of the historical event in order to minimize the location uncertainties. The coordinates of the center of the grid were chosen to be the epicentral coordinates of each event, the radius of the search area defined equal to $25 \mathrm{~km}$ and $10 \mathrm{~km}$ for the verification test. The gridding search space is defined equal to 1 $\mathrm{km}$ in both cases. For the reassessment of historical events the radius of the search area was preferred to be the one performing the most reliable location for the instrumental events.

\subsection{Statistical analysis}

For calculating earthquake recurrence times, first the long - term correlation between the values of each data set is investigated. This correlation is examined by calculating the Autocorrelation (ACF) and Partial Autocorrelation (PACF) functions. The Autocorrelation function examines the correlation between past and future values of time series (Eq. 6). Then the Partial Autocorrelation function can confirm the correlation detected by ACF (Eq. 7).

\section{Equation 6 - Autocorrelation function (ACF)}

$\rho_{k}=\sum_{i=1}^{N-k}\left(x_{i}-\bar{x}\right)\left(x_{i+k}-\bar{x}\right) / \sum_{i=1}^{N}\left(x_{i}-\bar{x}\right)^{2}$, where $N$ is the number of observations, $k$ is the number of the lags and $\bar{x}$ is the mean value of the sample.

\section{Equation 7 - Partial Autocorrelation function (PACF)}

$r_{k, k}=\left[\rho_{k}-\sum_{i=1}^{k-1} r_{k-1, j} \rho_{k-j}\right] /\left[1-\sum_{i=1}^{k-1} r_{k-1, j} \rho_{j}\right]$, where $r_{k, j}=r_{k-1, j}-r_{k, k} r_{k-1, j-1}$ for $j=1, \ldots, k$. If $k=1$ then $r_{1,1}=\rho_{1}$.

For the determination of earthquake recurrence time of each segment three statistical distributions were attempted in each data set, namely the Weibull, the inverse Gaussian and the lognormal with probability density functions (pdf) given from Equations 8, 9 and 10, respectively:

\section{Equation 8 - pdf of Weibull distribution}

$f(x \mid a, b)=(b / a)(x / a)^{b-1} \exp (-x / a)^{b}$, where $\alpha$ is the scale parameter and $b$ is the shape parameter.

\section{Equation 9 - pdf of Inverse Gaussian distribution}

$f(x \mid \mu, \lambda)=\left(\lambda / 2 \pi x^{3}\right)^{1 / 2} \exp \left\{-\lambda(x-\mu)^{2} / 2 \mu^{2} x\right\}$, where $\mu$ is the mean value and $\lambda$ is the shape parameter.

\section{Equation 10 - pdf of Lognormal distribution}

$f(x \mid \mu, \sigma)=(1 / x \sigma \sqrt{2 \pi}) \exp \left\{-(\ln (x)-\mu)^{2} / 2 \sigma^{2}\right\}$, where $\mu$ is the mean and $\sigma$ is the standard deviation of the random variable's natural logarithm.

The parameter estimation for each distribution was achieved by applying the Maximum Likelihood Estimation (MLE) method using the respective formulae (Johnson et al., 1994).

In order to compare the distributions and choose the best performing one in each data set the Anderson - Darling goodness of fit test (A - D test) is applied. The A - D test is implemented by calculating the distance, $A^{2}$, between the empirical cumulative distribution function (ecdf) and the cumulative distribution function (cdf) for each distribution applied to our data, according to the relation:

\section{Equation 11 - Distance between the empirical cdf and the distribution cdf}

$A^{2}=n \int_{-\infty}^{+\infty}\left[F_{n}(x)-F(x)\right]^{2} / F(x)[1-F(x)] d F(x)$, where $n$ is the number of observations, $F_{n}$ is the empirical cdf and $F$ is the cdf of the distribution which is under detection. 
The test then compares the factor $A^{2}$ with a critical value, $c$, under the null hypothesis that the data are distributed according to $F$. If the factor $A^{2}$ is less than or equal to the critical value, then the null hypothesis cannot be rejected.

The Akaike and Bayesian Information Criteria were also calculated. The difference between the two criteria is that BIC takes into account also the number, $n$, of the observations. This fact makes the penalty term of BIC larger than in AIC. The distribution, which displays the best performance to each data set, is the one with the minimum value of the criterion in both cases. The two criteria are given by the relations:

\section{Equation 12 - Akaike Information Criterion}

$A I C=-2 \ln (L)+2 k$, where $\ln (L)$ is the $\log -$ likelihood function and $k$ the number of parameters.

\section{Equation 13 - Bayesian Information Criterion}

$B I C=-2 \ln (L)+k \ln (n)$, where $\ln (L)$ denotes the $\log -$ likelihood function, $k$ the number of parameters and $n$ the number of observations.

The distribution that displays the best performance to the data and consequently better describes the earthquake recurrence time is used for the probability calculations of the next earthquake occurrence $T$ in fixed time intervals taking into account the time $T_{n}$ of the last earthquake according to the relation:

Equation 14 - Earthquake occurrence probabilities relation

$P\left(T_{n}+t \leq T \leq T_{n}+t+d t \mid T>T_{n}+d t\right)=\int_{T_{n}+t}^{T_{n}+t+d t} f(T) d t$

\section{Data}

\subsection{Earthquake catalogues}

Information on strong earthquakes in North Aegean Trough covers both historical and instrumental events and is provided by the historical catalogue of Papazachos and Papazachou (2003) and by the regional catalogue of instrumental seismicity of Geophysics Department of the Aristotle University of Thessaloniki (http://geophysics.geo.auth.gr/ss). From these two sources 40 events with $M \geq 6.0$ are found in the time interval between 360 BC and 2014 AD. The temporal distribution of these earthquakes evidence that there are missing events at least until 1300 AD. After 1300 AD the earthquakes seem to cluster in time with a mean rate equal to 5.1 events per century. Thus, earthquakes that occurred after $1300 \mathrm{AD}$ (34 events) are taken into account for the recurrence times determination after removing the two strong aftershocks of 1912 earthquake occurred in Ganos fault segment.

\subsection{Macroseismic data}

Macroseismic information is taken from the database of Papazachos et al. (1997) for the period 426 BC - 1995. For each historical event, reassessment is done only in the case when 3 or more macroseismic observations were available. This becomes feasible for 19 out of 34 events. For the 2014 earthquake in particular, the data is taken from the felt reports of the Euro - Mediterranean Seismological Center (http://emsc-csem.org).

\section{Data processing and results}

\subsection{Relocation of historical events}

In order to reassess and minimize the errors contained in the historical catalogue, the method described by the Equations 1 to 5 was applied firstly to six events of the instrumental period (1905, 
1912, 1975, 1982, 1983 and 2014) for evaluating the method using two different gridding search radius, of 25 and $10 \mathrm{~km}$, respectively. It is derived that using a radius equal to $10 \mathrm{~km}$, the epicentral coordinates are in better agreement with those of the instrumental catalogue. Thus, this value of the radius search was decided to be used for all historical events. The association of the relocated epicenters with the fault segments is feasible with good agreement except of the cases of 1659, 1766, and 1893. The disagreement is due to insufficient macroseismic observations and the initial coordinates are used. The relocated seismicity was divided into three distinctive clusters, corresponding to three fault segments, namely the North Aegean basin (13 events between 1366 and 1983 with 1.84 earthquakes per century), Gulf of Saros (12 events between 1511 and 2014 with 2.49 earthquakes per century) and Ganos (7 events between 1354 and 1912 with 1.19 earthquakes per century).

For each fault segment, a data set $T_{r j}(\mathrm{j}=1,2,3)$ was created and analyzed through the statistical distributions previously described. Each data set is composed by the time differences between successive earthquakes, $T_{i+1}-T_{i}$.

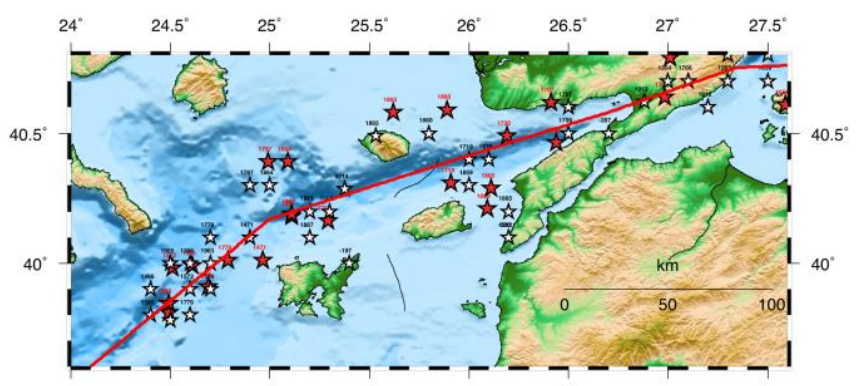

Figure 2 - Spatial distribution of strong $(M \geq 6.0)$ earthquakes at the initial (light grey asterisks) and relocated (red asterisks) position.

\subsection{Statistical analysis}

The $T_{r 1}, T_{r 2}$ and $T_{r 3}$ data samples (in years) for the North Aegean Basin, Gulf of Saros and Ganos segments consist of 12,11 and 6 observations, respectively, and are given below:

- North Aegean basin segment: $T_{r 1}=[90,15,93,8,13,191,3,18,67,41,77,1]$

- Gulf of Saros segment: $T_{r 2}=[158,50,11,26,9,94,1,27,6,82,39]$

- Ganos segment: $T_{r 3}=[83,222,48,59,99,47]$

For each data set, the Autocorrelation and Partial Autocorrelation functions were calculated and the results are shown in the diagrams of Figure 6. From both, the Autocorrelation and Partial Autocorrelation function values, it is evidenced that the correlations concerning the time intervals between successive earthquakes for each data set are statistically not significant, or in other words that there does not exist any correlation between them. The statistical distributions (Weibull, inverse Gaussian and lognormal) were applied to the three data sets and their parameters and $95 \%$ confidence intervals were estimated by the MLE method. Also, the log likelihood functions for each one of the distributions were recorded (Tables 1-3). It comes out that the confidence intervals in all cases are considerably of large range, probably due to the limited number of data in each sample. Especially for the inverse Gaussian distribution the confidence intervals in the three cases include negative and zero values due mainly to the limited number of observations in each sample, which are here meaningless (alternatively non - negativity constrains could be taken into account).

The Anderson - Darling test was applied to each sample in order to compare the distributions derived via the MLE parameter estimates and empirical cdf (Fig. 4); an estimated distribution is rejected if the distance, $A^{2}$ exceeds the critical value $c$ (Table 4 ). The results for the three samples manifest that the null hypothesis cannot be rejected for each distribution. The AIC and BIC values were calculated 
for each distribution and for the three data sets (Table 5). The distribution with the lower values of both criteria for the $T_{r 1}$ and $T_{r 2}$ samples is the Weibull and for the $T_{r 3}$ is the inverse Gaussian.

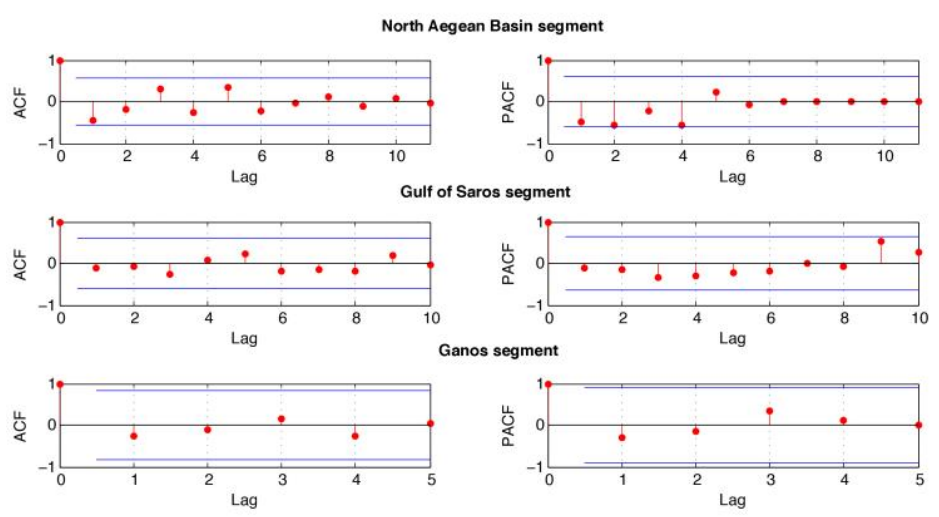

Figure 3 - Diagrams of Autocorrelation (left) and Partial Autocorrelation (right) functions of North Aegean basin (top), Gulf of Saros (middle) and Ganos (bottom) segment.

Table 1 - MLE parameters estimates, 95\% confidence intervals and log likelihood calculation for North Aegean basin segment.

\begin{tabular}{|c|c|c|c|}
\hline Distribution & Parameters & Conf. Intervals & $\log \mathbf{L}$ \\
& & & \\
\hline Weibull & $\alpha=47.289$ & {$[23.362,95.721]$} & -58.9969 \\
& $\mathrm{~b}=0.8449$ & {$[0.5355,1.3330]$} & \\
\hline Inverse Gaussian & $\mu=51.4167$ & {$[-22.422,125.254]$} & -61.3968 \\
& $\lambda=7.9814$ & {$[1.5950,14.3678]$} & \\
\hline Lognormal & $\mu=3.15735$ & {$[2.1639,4.1507]$} & -59.7785 \\
& $\sigma=1.56349$ & {$[1.1076,2.6546]$} & \\
\hline
\end{tabular}

Table 2 - MLE parameters estimates, $95 \%$ confidence intervals and log likelihood calculation for Gulf of Saros segment.

\begin{tabular}{|c|c|c|c|}
\hline Distribution & Parameters & Conf. Intervals & $\log \mathbf{L}$ \\
\hline Weibull & $\alpha=43.8778$ & {$[22.212,86.677]$} & -52.9769 \\
& $\mathrm{~b}=0.9140$ & {$[0.5702,1.4651]$} & \\
\hline Inverse Gaussian & $\mu=45.7273$ & {$[-16.568,108.023]$} & -55.5644 \\
& $\lambda=8.6043$ & {$[1.4134,15.7952]$} & \\
\hline Lognormal & $\mu=3.139$ & {$[2.1586,4.1194]$} & -53.7948 \\
& $\sigma=1.4593$ & {$[1.0196,2.5910]$} & \\
\hline
\end{tabular}

Table 3 - MLE parameters estimates, $95 \%$ confidence intervals and log likelihood calculation for Ganos segment.

\begin{tabular}{|c|c|c|c|}
\hline Distribution & Parameters & Conf. Intervals & $\log \mathbf{L}$ \\
\hline Weibull & $\alpha=105.4160$ & {$[63.8458,174.0520]$} & -31.9765 \\
& $\mathrm{~b}=1.6997$ & {$[0.9550,3.0253]$} & \\
\hline Inverse Gaussian & $\mu=93$ & {$[50.3465,135.6540]$} & -30.8999 \\
& $\lambda=283.063$ & {$[-37.2471,603.3740]$} & \\
\hline
\end{tabular}




\begin{tabular}{|c|c|c|c|}
\hline Lognormal & $\mu=4.3692$ & {$[3.7530,4.9854]$} & -31.0349 \\
& $\sigma=0.5872$ & {$[0.3665,1.4401]$} & \\
\hline
\end{tabular}
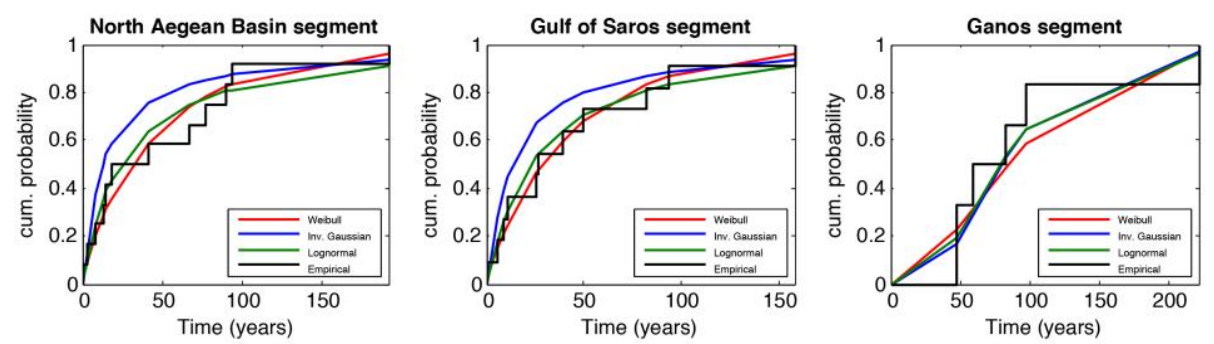

Figure 4 - Comparison of ecdf and estimated (theoretical) cdf for each distribution applied to the three data sets $\left(T_{r 1}\right.$ left, $T_{r 2}$ middle and $T_{r 3}$ right).

Table 5 - Results of Anderson - Darling test for the three data sets.

\begin{tabular}{|c|c|c|c|}
\hline \multirow{2}{*}{ Distribution } & $\boldsymbol{T}_{\boldsymbol{r}}$ & $\boldsymbol{T}_{\boldsymbol{r} 2}$ & $\boldsymbol{T}_{\boldsymbol{r} 3}$ \\
\cline { 2 - 4 } & $\boldsymbol{c}=\mathbf{2 . 5 0 8 4}$ & $\boldsymbol{c}=\mathbf{2 . 5 0 9 9}$ & $\boldsymbol{c}=\mathbf{2 . 5 2 4 8}$ \\
\hline & $\boldsymbol{A}^{2}$ & $\boldsymbol{A}^{2}$ & $\boldsymbol{A}^{\mathbf{2}}$ \\
\hline Weibull & 0.2572 & 0.1434 & 0.5443 \\
\hline Inverse Gaussian & 1.2883 & 1.2117 & 0.4347 \\
\hline Lognormal & 0.3242 & 0.2570 & 0.3861 \\
\hline
\end{tabular}

Table 1 - AIC and BIC values for the three data sets.

\begin{tabular}{|c|c|c|c|c|c|c|}
\hline \multirow{2}{*}{ Distribution } & \multicolumn{2}{|c|}{$T_{r 1}$} & \multicolumn{2}{c|}{$T_{r 2}$} & \multicolumn{2}{c|}{$T_{r 3}$} \\
\cline { 2 - 7 } & AIC & BIC & AIC & BIC & AIC & BIC \\
\hline Weibull & 121.99 & 122.96 & 109.95 & 110.75 & 67.95 & 67.54 \\
\hline Inverse Gaussian & 126.79 & 127.76 & 115.13 & 115.92 & 65.80 & 65.38 \\
\hline Lognormal & 123.56 & 124.53 & 111.59 & 112.38 & 66.07 & 65.65 \\
\hline
\end{tabular}

By combining the results of A - D test and the AIC and BIC criteria, we get that Weibull performs better than the other distributions in $T_{r 1}$ and $T_{r 2} \mathrm{~T}_{\mathrm{r} 2}$ data sets and inverse Gaussian in $T_{r 3}$ data set. In the two first cases (North Aegean and Gulf of Saros segments) we notice that the parameters b are less than 1 , which indicates that their hazard rates $h(x)=f(x) /[1-F(x)]$ decrease over time; this statement reflects the clustering behaviour of earthquake occurrence in this two segments, while they contain very small and very large time intervals. The Weibull distribution is then used for the estimation of the conditional occurrence probability of earthquakes with $M \geq 6.0$ for the next 30 years (after 2015) onto North Aegean Basin and Gulf of Saros segments and the inverse Gaussian probability density function on Ganos segment. For the North Aegean basin segment, where the last earthquake occurred in 1983, probabilities are low to intermediate for the next 30 years. For the Gulf of Saros segment, where the last earthquake occurred in 2014, probabilities of the next earthquake occurrence for next 30 years are intermediate and for the Ganos segment where the last strong earthquake occurred before over a century (103 years) the respective probabilities are intermediate. 

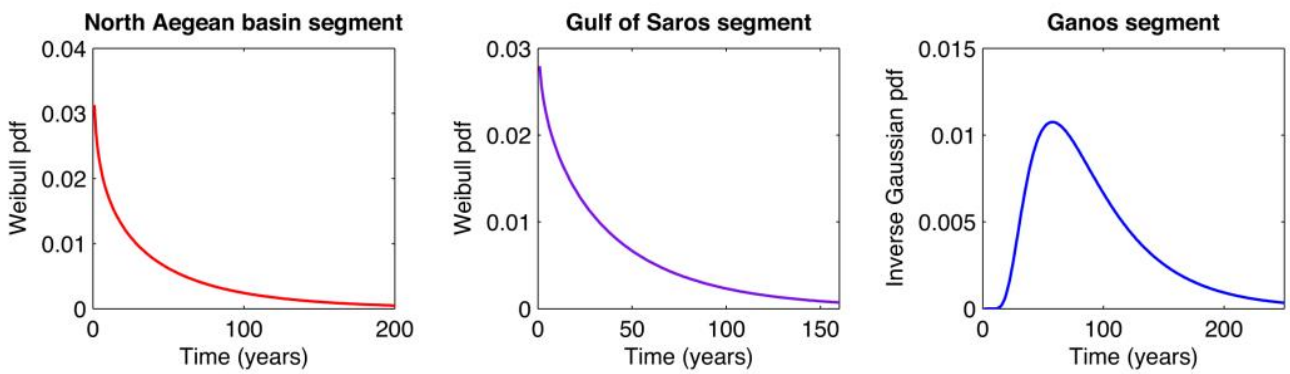

Figure 5 - Probability density functions for the North Aegean basin (left), Gulf of Saros (middle) and Ganos (right) segments.

Table 6 - Conditional occurrence probabilities for $M \geq 6.0$ earthquakes for the next 10, 20 and 30 years after 2015.

\begin{tabular}{|c|c|c|c|c|}
\hline Segment & Last Earthquake Occurrence & \multicolumn{3}{|c|}{ Time (in years after 2015) } \\
\hline & & $\mathbf{1 0}$ & $\mathbf{2 0}$ & $\mathbf{3 0}$ \\
\hline North Aegean Basin & 1983 & 0.1694 & 0.3055 & 0.4162 \\
\hline Gulf of Saros & 2014 & 0.2196 & 0.3780 & 0.4996 \\
\hline Ganos & 1912 & 0.1783 & 0.3265 & 0.4488 \\
\hline
\end{tabular}

\section{Conclusions}

Occurrence probabilities for earthquakes with $M \geq 6.0$ for each segment of North Aegean Trough were calculated after reassessment of their source parameters. The historical events relocation resulted reliable focal parameters for the most of them, except for three due to data shortage. Firstly the existence of long-term correlations between the values of each data with the ACF and PACF values was examined. These results revealed that there is not any correlation between the three samples. Then, Weibull, inverse Gaussian and lognormal distributions were applied to each data set. The confidence intervals of each parameter for the three distributions presents significantly great range, probably due to data shortage. Especially, the inverse Gaussian's 95\% confidence intervals include negative and zero values. Also, b parameter of Weibull distribution for the first two cases is less than the unity, which indicates that the earthquake occurrence in this segments have the tendency to cluster in time. The A - D goodness of fit test was applied for examining which of them performs better. In all cases the test does not reject (all) the distributions. Consequently, for further evaluation, the values of AIC and BIC criteria were calculated, and it is derived that the distribution with the best performance is Weibull for the North Aegean basin and Gulf of Saros segments and inverse Gaussian for Ganos segment. The conditional occurrence probabilities were thus calculated with the distribution, which fits better to each segment's data for the next 10, 20 and 30 years after 2015. The North Aegean basin segment exhibits a low to intermediate occurrence probability $(17 \%-41 \%)$ for the next 3 decades. The Gulf of Saros segment occurrence probabilities are not low (22\% - 50\%) although the last earthquake occurred 2014. The Ganos segment exhibits low to intermediate probability values $(17 \%-45 \%)$ for the next 30 years.

\section{Acknowledgments}

The authors are grateful to the reviewers for their detailed comments that were very useful for improving the manuscript. The maps were made using the Generic Mapping Tool 
(www.soest.hawaii.edu/gmt, Wessel and Smith 1998). Fault plane solutions data used in this paper came from http://www.ldeo.columbia. edu/ gcmt.

\section{References}

Abaimov, S.G., Turcotte, D.L., Shcherbakov, R., Rundle, J.B., Yakovlev, G., Goltz, C. and Newman, W.I, 2008. Earthquakes: Recurrence and interoccurrence Times, Pure Appl. Geophys., 165, 777-795, doi: /10.1007/s00024-008-0331-y.

Bakun, W.H. and Wentworth, C.M., 1997. Estimating earthquake location and magnitude from seismic intensity data, Bull. Seism. Soc. Am., 87, 1502-1521.

Hagiwara, Y., 1974. Probability of earthquake occurrence as obtained from a Weibull distribution analysis of crustal strain, Tectonophysics, 23, 318-323.

Jackson, D.D., Aki, K., Cornell, C.A., Dieterich, J.H., Henyey, T.L., Mahdyiar, M., Schwartz, D. and Ward, S.N., 1995. Seismic hazard in Southern California: Probable earthquakes, 1994 to 2024, Bull. Seism. Soc. Am., 85, 379-439.

Johnson, N.L., Kotz, S. and Balakrishnan, N., 1994. Continuous Univariate Distributions, vol.1, Wiley, 784 pp.

Kagan, Y.Y. and Knopoff, L., 1987. Random stress and earthquake statistics: Time dependence, Geoplys. J. R. Astron. Soc., 88, 723-731.

Matthews, V.M., Ellsworth, W.L. and Reasenberg, P.A., 2002. A Brownian Model for Recurrent Earthquakes, Bull. Seism. Soc. Am., 92, 2233-2250.

Nishenko, S. and Buland, R., 1987. A generic recurrence interval distribution for earthquake forecasting, Bull. Seism. Soc. Am., 77, 1382-1389.

Papazachos, B.C., Papaioannou, Ch.A., Papazachos, C.B. and Savvaidis, A.S., 1997a. Atlas of isos eismal maps for strong shallow earthquakes in Greece and surrounding area (426BC-1995), Ziti Publications, 192 pp.

Papazachos, B.C., Papadimitriou, E.E., Kiratzi, A.A., Papazachos, C.B. and Louvari, E.K., 1998. F ault plane solutions in the Aegean Sea and the surrounding area and their tectonic implicatio ns, Boll. Geof. teor. appl., 39, 199-218.

Papazachos, C. and Papaioannou, Ch., 1997. The macroseismic field of Balkan area, J. Seismol., 1, 181-201.

Papazachos, B.C. and Papazachou, C.C., 2003. The earthquakes of Greece, Ziti Publications, 304 pp.

Permanent Regional Seismological Network operated by the Aristotle University of Thessaloniki, doi: $10.7914 / \mathrm{SN} / \mathrm{HT}$.

Rikitake, T., 1976. Recurrence of great earthquakes at subduction zones, Tectonophysics, 35, 335-362.

Schwartz, D.P. and Coppersmith, K.J., 1984. Fault behavior and characteristic earthquakes: Examples from Wasatch and San Andreas fault zones, J. Geophys. Res., 89, 5681-5698.

Shimazaki, K., and Nakata, T., 1980. Time-predictable recurrence model for large earthquakes, Geophys. Res. Lett., 7, 279-282.

Wessel, P. and Smith, W. H.F., 1998. New, improved version of the generic mapping tools released, Trans. Am. Geophys. Union, 79, 579. 